\title{
DETC2013-12918
}

\section{DIMENSIONAL OPTIMIZATION OF A TWO-ARM ROBOT FOR SINGLE-SITE SURGERY OPERATIONS}

\author{
Christophe Drouin \\ University of Orleans \\ PRISME Laboratory \\ Bourges, Centre, FRANCE
}

\author{
AbolfazlPourghodrat \\ University of Nebraska-Lincoln \\ Mechanical \& Materials Engineering \\ Lincoln, NE, USA
}

\author{
Sylvain Miossec \\ University of Orleans \\ PRISME Laboratory \\ Bourges, Centre, FRANCE
}

\author{
Gérard Poisson \\ University of Orleans \\ PRISME Laboratory \\ Bourges, Centre, FRANCE
}

\author{
Carl A. Nelson \\ University of Nebraska-Lincoln \\ Mechanical \& Materials Engineering \\ Lincoln, NE, USA
}

\begin{abstract}
Unlike open surgery, minimally invasive surgery (MIS) involves small incisions through which instruments are passed to perform surgery. This technique is preferred since it reduces postoperative pain and recovery time. Laparoendoscopic single-site (LESS) surgery is the next step in MIS; a single incision is created instead of multiple access points for allowing the instruments to enter the peritoneal cavity. However, such minimally invasive techniques force the surgeon to perform more complex movements, hence the interest to use robotic systems. Design of robots for LESS is challenging to avoid collisions, reduce weight, and improve compactness while respecting the technical requirements (minimum forces, velocities). In this paper, we present the dimensional synthesis of a two-arm robot used for LESS. Each arm has a 2R-R-R architecture with link lengths optimized to respect the workspace constraints and maximize compactness while improving the performance in terms of forces and velocities (kinetostaticproperties).
\end{abstract}

\section{INTRODUCTION}

\section{MIS and LESS surgery}

Minimally invasive surgery (MIS) is performed through three to six tiny incisions, offering less pain and recovery time, shorter hospital stays, and improved cosmetic scarring as opposed to traditional open surgery. MIS has evolved to include laparoendoscopic single-site (LESS) surgery in which the surgeon operates through a single incision. To fully eliminate the external incision, natural orifice transluminal endoscopic surgery (NOTES) can be performed by accessing the peritoneal cavity with an endoscope passed through a natural orifice.

With the growing interest toward performing surgeries less invasively through a single incision or a natural orifice, more advanced instruments have been desired to overcome the difficulties associated with these types of surgical procedures, such as reduced dexterity and articulation. Robotic systems have come to address these difficulties and help implementation of MIS.The first generation of MIS robotic platforms are positioned above the patients and remotely controlled by a surgeon, manipulating laparoscopic tools. Examples of these types of externally driven robots include the da Vinci Surgical System [1], CoBRASurge [2], CURES [3], and Raven [4], among others.

Recently there has been a trend to miniaturize surgical robots and insert them inside the peritoneal cavity [5-12]. Fixed in vivo imaging robots were designed and built to assist surgeons with useful views of the surgical target [5]. With the help of motor-driven wheels, mobility was added to enable them not only in performing imaging but also exploring the abdominal cavity for tissue manipulation, dissection, cauterization, and drug delivery [6]. Traumatic traction on the organs as well as low force density of these robots motivated the development of dexterous miniature robot arms [7-11], whose mode of operation more closely mimics the methods used in laparoscopy.

An early design consisted of a two-arm tethered robot attached magnetically to the abdominal wall [7]. Tethered electronics as well as the compromise between the size of the robot and the dexterity and speed were drawbacks of this robot. 
Several versions of two-arm miniature in vivo robots with varying degrees of freedom, different linkages and joint designs, a range of topologies, and various methods of actuation have been designed, built and tested [8-12] in an attempt to increase the workspace, dexterity, force and torque density and simplicity of insertion along with further miniaturization. Figure 1 shows two representative examples of robots which have evolved in complexity; per-arm architectures include R-R-P, 2R-R-P, R-R-R-R-R-R, R-R-R-R, R-2R-R-R-R, 2R-2R-R, and 2R-R-R [8-11]. It should be noted that speeds and forces typical of various surgical procedures, as measured by the BlueDRAGON device and described in [13], have been used as design guidelines for most of these robots.

While a number of bimanual robots have been designed, built and tested, there has been little work on analysis to optimize the number of degree of freedom, joint type, link lengths and topology. The effect of different types of joints (revolute vs. prismatic) and link lengths on the workspace size of a NOTES bimanual robot has been discussed in [14].

This paper presents the dimensional synthesis of a miniature in vivo robot for LESS surgery (2R-R-R architecture) in order to extend previous work on this type of architecture [811]. We optimize the link lengths in the two arms to improve the compactness and kinetostatic performance of the robot while respecting workspace constraints.
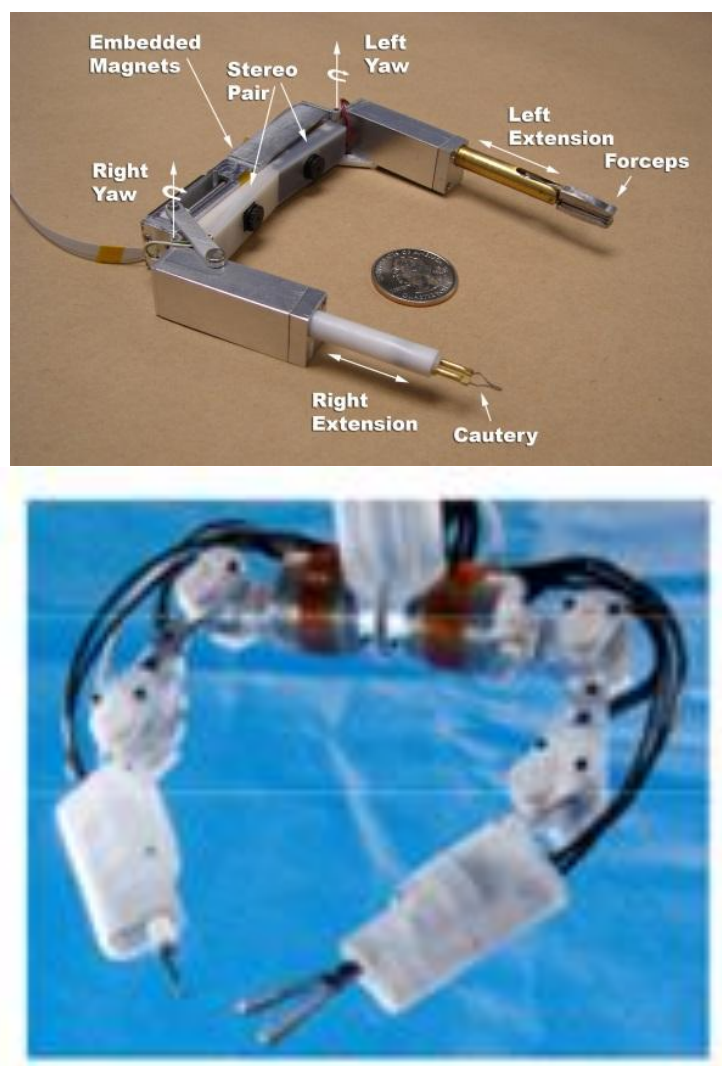

FIGURE 1.EVOLUTION OF THE LESS-ROBOT STRUCTURE

\section{ROBOT OVERVIEW}

\section{Robotdesign}

The robot having 2R-R-R architecture was chosen for optimization. Each link of this robot was initially dimensioned using a CAD approach in order to satisfy the workspaceconstraint and avoid collisions between the arms and the patient. The integration and choice of actuators was done witha combination of the CAD model and a mathematical analysis to the respect two main constraints: the kinetostatic requirements and the constraints of insertion. These two phases of design (dimensional synthesis and integration of actuators) were decoupled, although they share common objectives.

Indeed, the link dimensions have an impact on the robot compactness but also on its kinetostatic performance measures: the maximum transmissible forces and velocities at the end effector not only depend on the actuator characteristics but also on the relation between the input velocities/forces and the outputs, which is given by the Jacobian[15], which itself depends on the link lengths.

The experimentaldata (positions of the tools, minimal velocities and forces) gathered duringMIS operations [10] therefore offer a design guideline for simultaneously choosing the adapted actuators and the link lengths. Thekinetostatic requirements are difficult to respect for LESS robots, since there only exists one insertion trocar instead of several, constraining the size of actuators; furthermoreobtaining both sufficient forces and velocities are two antagonist objectives. Moreover, the links cannot be too longor too shortwithout compromisingeither good compactness or the workspace constraint.

The previous designs using CAD approaches have shown their limitations, withall these compromises to be made between kinetostatic performance, actuatorsize and link dimensions.

This paper aims to formulate and solve the problem of dimensional synthesisof the $2 \mathrm{R}-\mathrm{R}-\mathrm{R}$ topology with a mathematical approach in order to help the designer to face all these compromises. The actuators are already chosen which means that input velocities and forces are known.Output kinetostaticperformanceis maximized as well as compactness, while respecting the workspace constraints; a Pareto front is built considering compactness, minimal transmissible velocities and forces, offering preliminary information before a first CAD design.

\section{Structure of the robot}

The kinematic architecture of the robotis presented in Figure 2. The structureof the robot is composed of two arms, each one being dedicated to a specific task (e.g., grasping/stretching or cutting/cauterizing). They both have the same $2 \mathrm{R}-\mathrm{R}-\mathrm{R}$ architecture. 


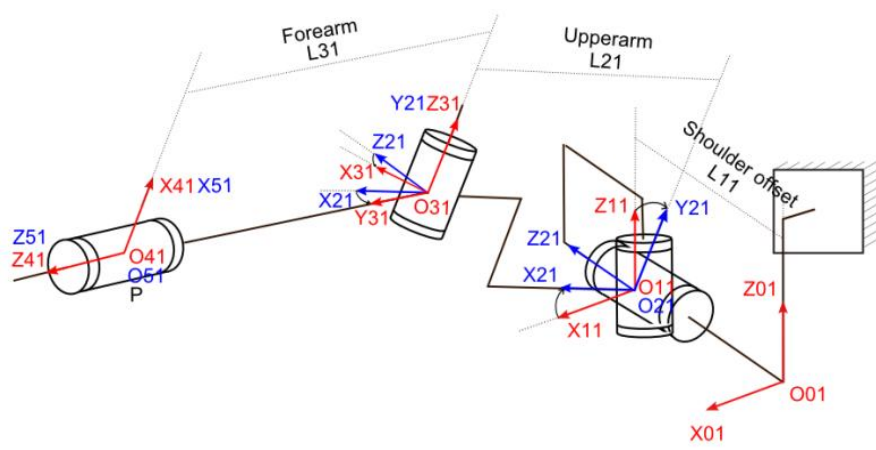

FIGURE 2.KINEMATIC ARCHITECTURE OF THE RIGHTARM (FOREARMS AND UPPER ARMS ARE TO BE OPTIMIZED)

The Denavit-Hartenbergparameters areindicated in Table 1using the non-modified conventionof Denavit-Hartenberg[15], with $j=1 . .2$, corresponding to the right and left arm respectively. $L_{11}$ definesthe shoulderoffset equal to $17.5 \mathrm{~mm}$ while $L_{21}$ and $L_{31}$ are the parameters to optimize. In the following treatment, $\sin (x)$ will be denoted $s_{x}$ and $\cos (x)$ as $c_{x}$.

TABLE 1.DENAVIT-HARTENBERG PARAMETERS

\begin{tabular}{|c|c|c|c|c|}
\hline Joint & 1 & 2 & 3 & 4 \\
\hline$\alpha_{i-1 j}$ & 0 & $\frac{\pi}{2}$ & $-\frac{\pi}{2}$ & $-\frac{\pi}{2}$ \\
\hline$a_{i-1 j}$ & $\begin{array}{c}a_{1 j} \\
=L_{1 j}\end{array}$ & 0 & $a_{2 j}=L_{2 j}$ & 0 \\
\hline$\theta_{i j}$ & $\theta_{1 j}$ & $\theta_{2 j}$ & $\theta_{3 j}$ & $\theta_{4 j}$ \\
\hline$r_{i j}$ & 0 & 0 & 0 & $\begin{array}{c}r_{1 j} \\
=L_{3 j}\end{array}$ \\
\hline $\begin{array}{c}\theta \text { (Figure 2, right } \\
\text { arm) }\end{array}$ & 0 & $\delta \theta_{21}$ & $-\frac{\pi}{2}+\delta \theta_{31}$ & $-\frac{\pi}{2}$ \\
\hline $\begin{array}{c}\text { Max. torque } \\
\text { (N.mm) }\end{array}$ & 1627.6 & 1627.6 & 245.8 & $47.3 / 264$ \\
\hline $\begin{array}{c}\text { Max. velocity } \\
\text { (rad/s) }\end{array}$ & 1.5 & 1.5 & 1.7 & $5.9 / 2.0$ \\
\hline
\end{tabular}

\section{Direct and inverse model}

The direct model enables us to write the relation between the joint motions and the end-effector position in a 3D Cartesian space, as follows (the direct/inverse kinematic model of the left arm will not be detailed, because of symmetry):

$$
\left[\begin{array}{l}
P X_{j} \\
P Y_{j} \\
P Z_{j}
\end{array}\right]=f\left(\left[\begin{array}{l}
\theta_{1 j} \\
\theta_{2 j} \\
\theta_{3 j}
\end{array}\right]\right)
$$

The model is given with the homogeneous DH transformation, with $j=1.2$ 2once again representing respectively the right and left arms:

$$
\begin{aligned}
\left(i-{ }^{1} T_{i}\right) j= & {\left[\begin{array}{cccc}
c_{\theta_{i j}} & -s_{\theta_{i j}} & 0 & a_{i-1 j} \\
s_{\theta_{i j}} c_{\alpha_{i-1 j}} & c_{\theta_{i j}} c_{\alpha_{i-1 j}} & -s_{\alpha_{i-1 j}} & -s_{\alpha_{i-1 j}} d_{i j} \\
s_{\theta_{i j}} s_{\alpha_{i-1 j}} & c_{\theta_{i j}} s_{\alpha_{i-1 j}} & c_{\alpha_{i-1 j}} & c_{\alpha_{i-j 1}} d_{i j} \\
0 & 0 & 0 & 1
\end{array}\right](2) } \\
& {\left[\begin{array}{c}
P X_{j} \\
P Y_{j} \\
P Z_{j}
\end{array}\right]=\left({ }^{0} T_{1}\right) j\left({ }^{1} T_{2}\right) j\left(2 T_{3}\right) j\left(3 T_{4}\right) j\left[\begin{array}{l}
0 \\
0 \\
0 \\
1
\end{array}\right](3) }
\end{aligned}
$$

For the rightarm, we will have:

$$
\left[\begin{array}{l}
P X_{1} \\
P Y_{1} \\
P Z_{1}
\end{array}\right]=\left[\begin{array}{c}
c_{\theta_{11}} c_{\theta_{21}}\left(L_{21}-L_{31} c_{\theta_{31}}\right)-L_{31} s_{\theta_{11}} c_{\theta_{31}} \\
s_{\theta_{11}} c_{\theta_{21}}\left(L_{21}-L_{31} c_{\theta_{31}}\right)-L_{31} c_{\theta_{11}} c_{\theta_{31}}-L_{11} \\
s_{\theta_{21}}\left(L_{21}-L_{31} s_{\theta_{31}}\right)
\end{array}\right]
$$

Equations of the inverse model are indicated below and not detailed for convenience:

$$
\begin{aligned}
& {\left[\begin{array}{l}
\theta_{11} \\
\theta_{21} \\
\theta_{31}
\end{array}\right]=g\left(\left[\begin{array}{l}
P X_{1} \\
P Y_{1} \\
P Z_{1}
\end{array}\right]\right)} \\
& {\left[\begin{array}{l}
\theta_{11} \\
\theta_{21} \\
\theta_{31}
\end{array}\right]=} \\
& {\left[\begin{array}{c}
2 \operatorname{atan} 2\left(\left(P Y_{1}+L_{11} \pm \sqrt{P X_{1}^{2}+\left(L_{11}+P Y_{1}\right)^{2}-e_{1}}\right),\left(P X_{1}+\sqrt{e_{1}}\right)\right) \\
\operatorname{atan2}\left(P Z_{1},\left(c_{\theta_{11}} P X_{1}+s_{\theta_{11}}\left(P Y_{1}+L_{11}\right)\right)\right) \\
\text { 2atan } 2\left(\left(L_{21}-c_{\theta_{21}}\right)\left(c_{\theta_{11}} P X_{1}+s_{\theta_{11}}\left(P Y_{1}+L_{11}\right)\right)-s_{\theta_{21}} P Z_{1}, s_{\theta_{11}} P X_{1}+c_{\theta_{11}}\left(P Y_{1}+L_{11}\right)\right.
\end{array}\right]}
\end{aligned}
$$

with:

$$
\begin{gathered}
e_{1}=d_{1}{ }^{2}-P Z_{1}^{2} \\
d_{1}=\frac{1}{2}\left(L_{21}-\frac{L_{31}^{2}}{L_{21}}+\left(P X_{1}^{2}+\left(L_{11}+P Y_{1}\right)^{2}+P Z_{1}^{2}\right) / L_{21}\right)
\end{gathered}
$$

The study of the inverse model shows there is not a solution in certain cases. These cases correspond to the unreachable positions of the robot, i.e., they indicate the boundaries of its workspace. In particular, it can be proven that when we have $e_{1}<0$, this corresponds to unreachable positions. These boundaries or unreachable zones are described in the following subsection. It should also be mentioned that for a given position, two solutions are possible, as shown by the " \pm " sign in the first term.

\section{Jacobian and singularities}

The direct instantaneous kinematic model gives the relation between the joint angular velocities and the end-effector velocities: 


$$
\dot{X}_{j}=J_{j} \dot{Q}_{j} \text {, with } X_{j}=\left[\begin{array}{c}
P X_{j} \\
P Y_{j} \\
P Z_{j}
\end{array}\right] \text { and } Q=\left[\begin{array}{c}
\theta_{1 j} \\
\theta_{2 j} \\
\theta_{3 j}
\end{array}\right]
$$

with $J_{1}$ detailed as follows:

$$
\begin{aligned}
& J_{1}= \\
& {\left[-\left(L_{21} \overrightarrow{x_{21}}+L_{31} \overrightarrow{y_{31}}\right) \wedge \overrightarrow{z_{11}}-\left(L_{21} \overrightarrow{x_{21}}+L_{31} \overrightarrow{y_{31}}\right) \wedge \overrightarrow{z_{21}}-L_{31} \overrightarrow{x_{31}}\right]}
\end{aligned}
$$

The study of $J_{1}$ enables us to find conditions of singularities, i.e., configurations of the robot when $\operatorname{det}\left(J_{1}\right)=0$. We have, with $s_{x}=\sin (x)$ and $c_{x}=\cos (x)$ :

$$
\begin{gathered}
\overrightarrow{J_{1}}(1)=\left[\begin{array}{c}
-L_{31}\left(c_{\theta_{11}} c_{\theta_{31}}-c_{\theta_{21}} s_{\theta_{11}} s_{\theta_{31}}\right)-L_{21} c_{\theta_{21}} s_{\theta_{11}} \\
-L_{31}\left(s_{\theta_{11}} c_{\theta_{31}}+c_{\theta_{21}} c_{\theta_{11}} s_{\theta_{31}}\right)+L_{21} c_{\theta_{11}} c_{\theta_{21}}
\end{array}\right] \\
0 \\
\overrightarrow{J_{1}}(2)=\left[\begin{array}{c}
-c_{\theta_{11}}\left(L_{21} s_{\theta_{21}}-L_{31} s_{\theta_{21}} s_{\theta_{31}}\right) \\
-s_{\theta_{11}}\left(L_{21} s_{\theta_{21}}-L_{31} s_{\theta_{21}} s_{\theta_{31}}\right) \\
L_{21} c_{\theta_{21}}-L_{31} c_{\theta_{21}} s_{\theta_{31}}
\end{array}\right] \\
\overrightarrow{J_{1}}(3)=\left[\begin{array}{c}
-L_{31}\left(c_{\theta_{11}} c_{\theta_{21}} c_{\theta_{31}}-s_{\theta_{11}} s_{\theta_{31}}\right) \\
-L_{31}\left(c_{\theta_{11}} s_{\theta_{31}}+s_{\theta_{11}} c_{\theta_{31}} c_{\theta_{21}}\right) \\
-L_{31} c_{\theta_{31}} s_{\theta_{21}}
\end{array}\right]
\end{gathered}
$$

The study of these three vectors shows that threesingularity cases can be distinguished:

- $\theta_{31}=\frac{\pi}{2}$ or $-\frac{\pi}{2}$

- $\theta_{21}=\frac{\pi}{2}$ or $-\frac{\pi}{2}$

- $\theta_{21}=0$ and $L_{21}=0$

These casesare represented in Figures3-5.

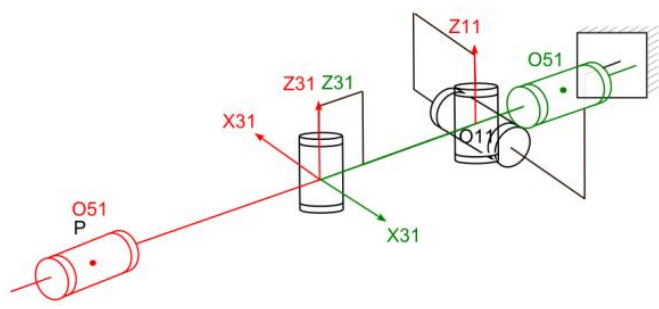

FIGURE 3.FIRST SINGULARITY CASE WHEN $\theta_{31}=\frac{\pi}{2}$ (GREEN) OR $\theta_{31}=-\frac{\pi}{2}$ (RED)

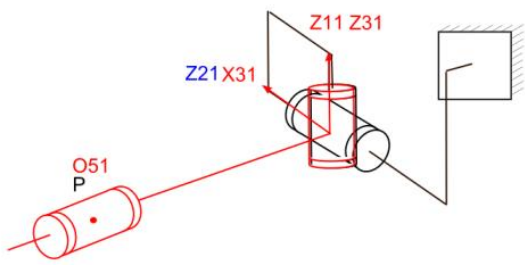

FIGURE 4.SECOND SINGULARITY CASE WHEN $\theta_{21}=$ $0 \mathrm{ANDL}_{21}=0$

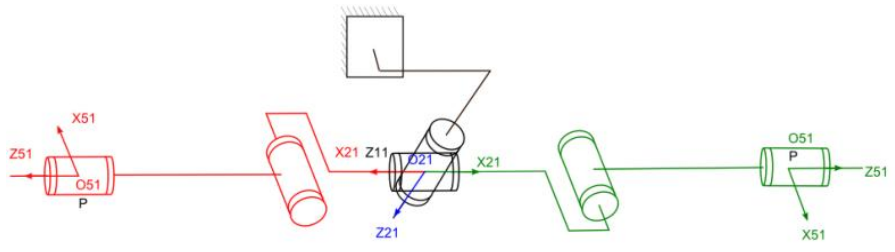

FIGURE 5.THIRD SINGULARITY CASE WHEN $\theta_{21}=\frac{\pi}{2}$ (RED) OR $\theta_{21}=-\frac{\pi}{2}$ (GREEN)

In the first case, a singularity appears when the arms are aligned; it indicates the exterior and interior boundaries of the workspace, giving a hollow sphere (red-brown and rosy-brown in Figure 6). The second case is physically infeasible $\left(L_{21}=0\right)$. The third case indicates there is an unreachable volume in the sphere, which forms two spherical voids, indicated in sandybrownin Figure 6.

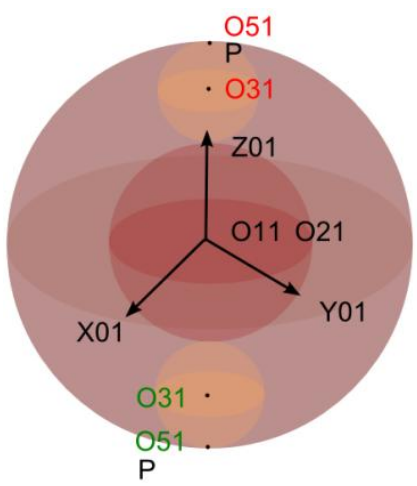

\section{FIGURE 6.SHAPE OF THE WORKSPACE}

The workspace isthereforea hollowsphere with inner diameter equal to $\left|L_{2 j}-L_{3 j}\right|$ and external diameter $L_{2 j}+L_{3 j}$, with two spherical voids of radius $L_{3 j}$. Other voids in the workspacedepend on motion limitsof the joints and on collisions of the robot torso and the other arm, which are not considered in this paper.

\section{OPTIMIZATION}

This section presents the dimensional synthesis of the robot; the parameters sought to optimize the compactness, transmissible velocities and forces are the lengths $L_{2 j}$ and $L_{3 j}$. They form the design vector $X=\left[L_{2 j} ; L_{3 j}\right]$.

\section{Data}

Experimental dataare presented first in [10]. Depending on the type of operation and the variability of operational volume between different peritoneal cavities of patients, the tools must reach a given set of points, which are different for the two arms. Table 2summarizes all this information. 
TABLE 2.DATA FOR THE RIGHT AND LEFT ARM

\begin{tabular}{|c|c|c|c|}
\hline $\begin{array}{c}\text { Right arm } \\
\text { position (mm) }\end{array}$ & $X_{5 j}$ & $Y_{5 j}$ & $Z_{5 j}$ \\
\hline $\begin{array}{c}\text { Open } \\
\text { cholecystectomy }\end{array}$ & {$[40 ; 100]$} & {$[0 ; 80]$} & {$[-150 ; 50]$} \\
\hline $\begin{array}{c}\text { In vivo } \\
\text { Colectomy }\end{array}$ & {$[0 ; 120]$} & {$[-80 ; 0]$} & {$[-130 ; 0]$} \\
\hline $\begin{array}{c}\text { Left arm position } \\
\text { (mm) }\end{array}$ & $X_{5 j}$ & $Y_{5 j}$ & $Z_{5 j}$ \\
\hline $\begin{array}{c}\text { Open } \\
\text { cholecystectomy }\end{array}$ & {$[10 ; 50]$} & {$[25 ; 100]$} & {$[-170 ; 0]$} \\
\hline $\begin{array}{c}\text { In vivo } \\
\text { Colectomy }\end{array}$ & {$[5 ; 60]$} & {$[-60 ; 0]$} & {$[-125 ; 25]$} \\
\hline
\end{tabular}

The shape of the set is approximated by an ellipsoid, whose semi-major and semi-minor axes are determined based on the minimum and maximum boundaries of the aforementioned data. Because the data are different, we consider two different ellipsoids, corresponding to the two arms.

Moreover, since the robot can be roughly positioned by the physician before operating, the ellipsoids are shifted on the $\mathrm{Z}_{0 \mathrm{j}}$ and $\mathrm{Y}_{0 \mathrm{j}}$ axes (with the same offset), to keep them as symmetric as possible on the $\mathrm{X}_{0 \mathrm{j}} \mathrm{Y}_{0 \mathrm{j}}$ and $\mathrm{X}_{0 \mathrm{j}} \mathrm{Z}_{0 \mathrm{j}}$ planes. The ellipsoids are also shifted on the $X_{0 j}$ axis from $O_{0 j}$ (with the same offset) for both arms, to provide the required offset from the $\mathrm{Z}_{0 \mathrm{j}}$ axis.

\section{Constraints}

The first type of constraint concerns the limits of the workspace. Its shape has been discussed in a previous section. We define $S_{j}$ as the two tool-tip position sets, with $S_{j}=$ $\left\{M_{1 j} ; M_{2 j} \ldots ; M_{n j}\right\}$ and we define $D_{S j}$ the two distances sets corresponding to the distances from $O_{1 j}$ to the point $M_{n j}$ ( $\mathrm{n}=$ number of points), with $D_{S_{j}}=\left\{d\left(M_{1 j}\right) ; d\left(M_{2 j}\right) \ldots ; d\left(M_{n j}\right)\right\}$ and we define $D^{\prime} s_{j}=\left\{d^{\prime}\left(M_{1 j}\right) ; d^{\prime}\left(M_{2 j}\right) \ldots ; d^{\prime}\left(M_{n j}\right)\right\}$ the set corresponding to the distances from $O_{4 j}$ to the point $M_{n j}$. Assuming that $L_{3 j}<$ $L_{2 j}$, we must have $L_{2 j}-L_{3 j}<\min \left(D_{S_{j}}\right)$ and $L_{2 j}+L_{3 j}>$ $\max \left(D_{S_{j}}\right)$ to reach all the points in the hollow sphere; we also must have min $\left(D^{\prime}{ }_{S_{j}}\right)>L_{3 j}$ to reach the points outside of the spherical voids. These three workspace constraints will be written as $C_{W_{1 j}}, C_{W_{2 j}}$ and $C_{W_{3 j}}$.

The second type of constraint concerns the collisions between the arms during operation. When $\mathrm{Z}_{3 \mathrm{j}}, \mathrm{Z}_{1 \mathrm{j}} j=1 . .2$ are perpendicular to the same plane, this presents a $2 \mathrm{D}$ collisionscenario. In this case, collisions are avoided if $\theta_{11} \in$ $\left[-180^{\circ} ; 0^{\circ}\right]$ and $\theta_{12} \in\left[0^{\circ} ; 180^{\circ}\right]$. To respect this constraint, we must consider only different solutions to the inverse model of both arms. In a 3D case such interferences can be avoided; yet, we will simplify the problem by considering the same limits on $\theta_{11}$ and $\theta_{12}$ as if we were in the $2 \mathrm{D}$ case.
It is noteworthy here that the data are consistent, in other words, no collision occurs between the two endeffectors.

\section{Performance criteria}

One of the major challenges concerning the design of in vivo robots concerns the improvement of kinetostatic performance. Classical indices like the dexterity [16] or the manipulability [17] have been used to estimate kinetostatic performance; however, they must be used carefully. To the extent that performance is related to precision and isotropy, they are well adapted. They can also be used to identify singularities, but they are not necessarily suitable to evaluate proximity to singularities.

Indeed, these indices do not take into account the actuator characteristics [18]: the dexterity is evaluated with the norm of input velocities equal to one, although this is not physically the case, and this can affect the interpretation of the performance measure.

Moreover, it is well-known that a robot is close to a singularity when the end-effector transmissible velocity reaches zero in a particular direction; it is therefore useless to evaluate the ratio of the minimal and maximal velocities to evaluate the proximity to a singularity, but only the minimal velocity itself.

Taking into account the actuator characteristics has another impact concerning the duality between kinematics and statics. This must be considered carefully since the maximum eigenvalue of the Jacobian does not represent the minimal exerted force in a particular direction.

For our application, improvement of the kinetostatic performance of the robot means increasing the end-effector velocity and force for any given configuration of the robot. The minimal velocities to increase are located near the worst cases, i.e., the singular configurations of the robot described earlier; isotropy is not sought here. Based on the work of Briot [19], we maximize thevaluesmin $\left(k_{V j}^{\min }\right)_{S_{j}}$ and $\min \left(k_{F j}^{\min }\right)_{S_{j}}$, defined as the lowest values of transmission factors in velocity and forces on the sets $S_{j}$.These transmission factors are defined as the minimum transmissible velocity along a particular direction at the end effector for a given configuration $S_{j}$ and the minimum transmissible force along a particular direction at the end effector for a given configuration $S_{j}$. Physically, $\min _{S_{j}}\left(k_{V j}{ }^{\min }\right)$ represents the lowest velocity in a particular direction for any configuration, equal to zero when we meet a singularity. $k_{V j}{ }^{\min }$ represents the minimal velocity along a particular direction. In one direction at the end effector, for a given point $S_{j}$, we have:

${k_{V j}}^{\min }=\min \left(k_{i j k}{ }^{(m)}\right)=\sqrt{J_{2 j}^{T} J_{2 j}-\left(J_{2 j}^{T} J_{1 j}\right)\left(J_{1 j}^{T} J_{1 j}\right)^{-1}\left(J_{1 j}^{T} J_{2 j}\right)}$

where $J_{2 j}=\left[\begin{array}{ll}I_{g j} & I_{h j}\end{array}\right], J_{1 j}=(-1)^{m} I_{i j}, \quad$ for $g, h, i=1,2,3$, $g \neq h \neq i, m=1$ or 2, with $\left[\begin{array}{lll}I_{1 j} & I_{2 j} & I_{3 j}\end{array}\right]=$

$J_{j} \operatorname{diag}\left(\dot{\theta}_{k j \text { max }}\right) \cdot \dot{\theta}_{k j \text { max }}$ represents the maximal velocity of the $k^{\text {th }}$ actuator of the $j^{\text {th }}$ arm. 


$$
k_{v j}{ }^{\max }=\max _{l}\left(\left\|J_{j}(\mathrm{q}) e_{l}\right\|\right), \text { for } l=1 \text { to } 4,
$$

where $e_{1}=[1 ;-1 ; 1]^{T} e_{2}=[1 ; 1 ; 1]^{T} e_{3}=[1 ; 1 ;-1]^{T} e_{4}=$ $[1 ;-1 ;-1]^{T}$.

The same evaluation of $\left(k_{F j}{ }^{\text {min }}\right)_{S_{j}}$ is done by replacing $J_{j} \operatorname{diag}\left(\dot{\theta}_{k j \text { max }}\right)$ with $J_{j}^{-T} \operatorname{diag}\left(T_{k j \text { max }}\right)$, where $T_{k j \max }$ represents the maximal torque of the $k^{\text {th }}$ actuator of the $j^{\text {th }}$ arm.

To summarize, the two kinetostaticperformance criteria $P_{\text {Velocity }}$ and $P_{\text {Force }}$ will be written as follows:

$$
\begin{gathered}
\mathrm{P}_{\text {Velocit } y_{j}}=\min \left(k_{V j}^{\text {min }}\right)_{S_{j}} \\
\mathrm{P}_{\text {Forc } e_{j}}=\min \left(k_{F j}^{\text {min }}\right)_{S_{j}}
\end{gathered}
$$

Another criterion to optimize is the compactness; this criterion has been defined as the sum of lengths $L_{2 j}$ and $L_{3 j}$, as follows:

$$
P_{\text {Compactnes } s_{j}}=\frac{L_{2 j}+L_{3 j}}{2}
$$

\section{The objective function}

A gradient-based method was used to find the optimal parameters $\left(L_{2 j}, L_{3 j}\right)$ satisfying the constraints and giving the optimal solutions between compactness and kinematic performance.The problem wasformulated as follows:

$$
\begin{gathered}
\min f(X)_{j}=\beta\left[(1-\alpha)\left(P_{\text {Compactnes } s_{j}}\right)-\alpha N\left(\mathrm{P}_{\text {Velocit } y_{j}}\right)\right] \\
+(1-\beta)\left(\mathrm{P}_{\text {Forc } e_{j}}\right) \\
\text { subject to } C_{W_{k j}}(V)>0, k=1 \ldots 3
\end{gathered}
$$

with $N$ a normalization coefficient, $\alpha$ and $\beta$ two weighting coefficients between the three criteria, $\alpha, \beta=[0 ; 1]$ to compute the Pareto surface as explained below.

\section{Results}

Results of optimization are represented in Figures7-9for only the right arm. Corresponding lengths for the left and right arm critical points are detailed in Table 3.
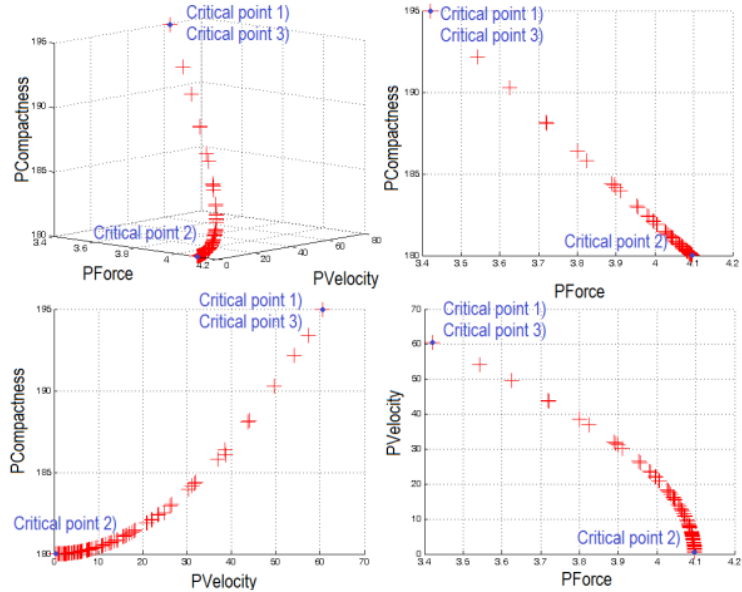

FIGURE 7.PARETO CURVESFOR THE RIGHT ARM $\left(\mathrm{P}_{\text {Velocity }}\right.$ in $\mathrm{mm} / \mathrm{s}, \mathrm{P}_{\text {Force }}$ in $\mathrm{N}$ and $P_{\text {Compactness }}$ in $\mathrm{mm}$ ). Critical points 1) and 3) are mingled

Each point is an optimal solution $\left(L_{2 j}, L_{3 j}\right)$, obtained by varying $\alpha$ and $\beta$. Actuator characteristics are given in Table 1 .

Table 3 shows the various results: it was indeed expected that optimized links lengths depend on their respective workspace ellipsoids. Interpretation of the results for the left arm is similar to the right arm; hence, only the right-arm results will be presented.
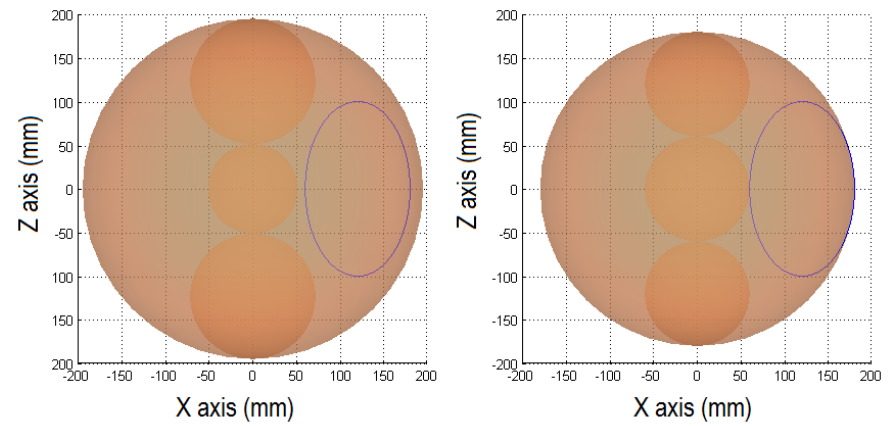

FIGURE 8.ELLIPSOID AND WORKSPACES FOR THE CRITICAL POINTS 1), 3) (left) AND 2) (right) OF THE PARETO SOLUTION
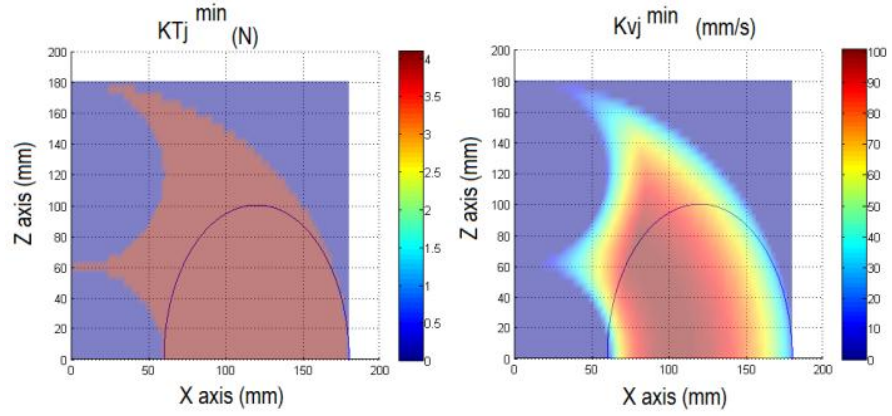

FIGURE 9. EVOLUTION OF $k_{V j}{ }^{\text {min }}$ AND $k_{T j}{ }^{\text {min }}$ FOR CRITICAL POINT 2)

TABLE 3. RESULTS FOR THE RIGHT AND LEFT ARM 


\begin{tabular}{|c|c|c|c|c|}
\hline & \multicolumn{2}{|c|}{ Right arm } & \multicolumn{2}{c|}{ Left arm } \\
\hline & $\begin{array}{c}\mathrm{P}_{\text {Velocity }} \\
\text { minimized }\end{array}$ & $\begin{array}{c}\mathrm{P}_{\text {Velocity }} \\
\text { maximized }\end{array}$ & $\begin{array}{c}\mathrm{P}_{\text {Velocity }} \\
\text { minimized }\end{array}$ & $\begin{array}{c}\mathrm{P}_{\text {Velocity }} \\
\text { maximized }\end{array}$ \\
\hline $\begin{array}{c}\left(L_{2 j}, L_{3 j}\right) \\
(\mathrm{mm})\end{array}$ & $(120 ; 60)$ & $(123.14 ; 71.82)$ & $(101.29 ; 36.10)$ & $(111.12 ; 63.51)$ \\
\hline $\begin{array}{c}\mathrm{P}_{\text {Compactness }} \\
(\mathrm{mm})\end{array}$ & 180 & 194.94 & 137.39 & 174.64 \\
\hline $\begin{array}{c}\mathrm{P}_{\text {Velocity }} \\
(\mathrm{mm} / \mathrm{s})\end{array}$ & 0.46 & 60.54 & 0.64 & 85.25 \\
\hline $\begin{array}{c}\mathrm{P}_{\text {Force }} \\
(\mathrm{N})\end{array}$ & 4.09 & 3.42 & 6.80 & 3.87 \\
\hline
\end{tabular}

In Figure 8, one can see that $\mathrm{P}_{\text {Compactness }}$ and $\mathrm{P}_{\text {Force }}$ are not opposite criteria: for some given torque for the three joints, increasing the lengths of the two links $\left(L_{2 j}, L_{3 j}\right)$ would reduce the effective force at the endeffector. Moreover, $\mathrm{P}_{\text {Compactness }}$ does not become infinite to improve $\mathrm{P}_{\text {Velocity }}$. For a certain value of $X=\left(L_{2 j}, L_{3 j}\right)$, it would be unnecessary to improve the lengths to improve $\mathrm{P}_{\text {Velocity }}$, since the two links would come closer to the singularity case where $\theta_{31}=-\pi / 2$, to reach the extrema points of the desirable volume. Furthermore, increasing $L_{2 j}$ is constrained by the fact that the spherical void would touch the ellipsoid. Then, Figure 10 on left side shows that $\mathrm{P}_{\text {Force }}$ is constant across the robot workspace.For the corresponding value of $X$ in this case, joint number 3 is the most ineffective joint in every configuration across the robot workspace. This means this actuator is under-dimensioned, and joint number 1 is over-dimensioned; in particular, the gear ratio of joint 1 could be increased and the gear ratio of joint 3 could be decreased.

The critical points (Figure 7) are the points where we have 1) the maximum of $\left.P_{\text {Velocity }}, 2\right)$ the minimum of $P_{\text {Velocity }}$ and 3) the compromise between $\mathrm{P}_{\text {Velocity }}$ and $\mathrm{P}_{\text {Compactness }}$. For the latter, we are close to having opposite relative variations between the two criteria as defined in [20], when $\mathrm{dP}_{\text {Compactness }} /$ $\mathrm{P}_{\text {Compactness }}=-d \mathrm{P}_{\text {Velocity }} / \mathrm{P}_{\text {Velocity }}$. Based on this definition, theoretically by a small variation along the Pareto front that will improve one criterion by $10 \%$, it will degrade the other criterion by $10 \%$. The results show this point c) is the same as for case a): it means it is useless to improve the compactness since it highly reduces the velocity; however, from the physician's point of view, it could be relevant to seek for the best compactness.

\section{CONCLUSIONS AND FUTURE WORK}

In this paper, the dimensional synthesis of a two-arm robot has been presented, for minimally invasive surgery operations. The link lengths have been optimized to find a compromise between kinetostatic performance and compactness, under constraints of workspace volume and shape. The evaluation of kinetostatic performance has been performed, taking into account the actuator characteristics. Results show the variation of minimal effective velocity according to compactness, the constancy of the minimum effective force and the influence of the spherical void on the results. This highlights the need to re-dimension the actuators and gear ratios for better performance.

The singularities in the workspace interfere with these outcomes in terms of feasible Pareto solutions, indicating the need for a topological optimization. Our future work will therefore concern two topics: 1) optimizing the topology of the robot considering the shape and volume of data and 2) coupling the robot dimensional synthesis with the choice of the actuator characteristicsunder constraints of integration. Another topic of improvement will concern the collisions of the arms in the 3D case.

\section{ACKNOWLEDGMENTS}

The authors gratefully acknowledge Prof. S. Farritor and his research assistants for allowing access to their data, information, and images related to their robot designs.

\section{REFERENCES}

[1] Intuitive Surgical, Inc., "the da Vinci Surgical System," accessed Dec. 15, 2012, < www.intuitivesurgical.com/products/davinci_surgical_syst $\mathrm{em} />$.

[2] Nelson, C. A., Zhang, X., Shah, B. C., Goede, M. R. and Oleynikov, D., 2010, "Multipurpose surgical robot as laparoscope assistant," J. Surgical Endoscopy, 24(7), pp. 1528-1532.

[3] Kim, S. K., Shin, W. H., Ko, S. Y., Kim, J. and Kwon, D. S., 2008, "Design of a Compact 5-DOF Surgical Robot of a Spherical Mechanism: CURES," Proc. IEEE/ASME International Conference on Advanced Intelligent Mechatronics, Xi'an, China, pp. 990-995.

[4] Lum, M., Friedman, D., Sankaranarayanan, G., King, H., Fodero, K., Leuschke, R., Hannaford, B. and Rosen, J., 2009, "The RAVEN: Design and Validation of a Telesurgery System," The International Journal of Robotics Research, 28(9), pp. 1183-1197.

[5] Rentschler, M., Hadzialic, A., Dumpert, J., Platt S.R., Farritor, S., and Oleynikov, D., 2004, "In Vivo Robots for Laparoscopic Surgery," Studies in Health Technology and Informatics, 98: 316-322.

[6] Hawks, J., 2010, "Improved mobile wireless in vivo surgical robots: Modular design, experimental results, and analysis" Ph.D. thesis, http://www. digitalcommons.unl.edu/mechengdiss/17/.

[7] Dumpert, J., Wood, N.A., Redden, L., Visty, A.Q., Farritor, S., Varnell, B., Oleynikov, D., and Lehman, A.C., 2009, "Natural orifice cholecystectomy using a miniature robot," Surgical Endoscopy, vol. 23, pp. 260-266.

[8] Lehman, A.C., Wood N.A., Farritor, S., Goede, M.R., and Oleynikov, D., 2011, "Dexterous miniature robot for advanced minimally invasive surgery," Surgical Endoscopy, vol. 25, pp. 119-123.

[9] Piccigallo, M., Scarfogliero,U., Quaglia, C., and Petroni, G., 2010, "Design of a Novel Bimanual Robotic System for 
Single-Port Laparoscopy," IEEE/ASME Transactions on Mechatronics, vol. 15, No. 6, pp. 871-878.

[10] Wortman, T. D., Strabala, K. W., Lehman, A. C., Farritor, S. M. and Oleynikov, D., 2011, "Laparoendoscopic singlesite surgery using a multi-functional miniature in vivo robot," International Journal of Medical Robotics and Computer Assisted Surgery, 7(1), pp. 17-21.

[11] Farritor, S., 2013, personal communication, January 2013.

[12] Pourghodrat, A., Nelson, C. A., and Midday, J., 2011, "Pneumatic miniature robot for laparoendoscopic single incision surgery," ASME Intl. Design Engineering Technical Conf., paper DETC2012-70454.

[13] Brown, J.D., Chang, L., Sinanan, M.N., and Hannaford, B., 2009, "Generalized approach for modeling minimally invasive surgery as a stochastic process using a discrete Markov model." IEEE Transactions on Biomedical Engineering, vol. 53, pp. 399-413.

[14] Seow, C. M., Chin, W. J., and Nelson, C. A., 2011, "Robot kinematic design studies for natural orifice surgery," ASME Intl. Design Engineering Technical Conf., paper DETC2011-47961.

[15] Denavit, J., Hartenberg, R., 1955. "A kinematic notation for lower-pair mechanisms based on matrices,"ASME Journal of Applied Mechanics,vol. 23, pp. 215-221.

[16] Gosselin, C., and Angeles, J., 1989, "The Optimum Design of a Spherical Three-Degree-of-Freedom Parallel Manipulator," ASME Journal of Mechanisms, Transmissions, and Automation in Design, Vol. 111, No. 2, pp. 202-207.

[17] Yoshikawa, T., 1985, "Manipulability of Robotic Mechanisms," Intl. J. of Robotics Research, vol. 4, no. 2, pp. 3-9.

[18] Merlet, J.-P., 2006, "Jacobian, manipulability, condition number, and accuracy of parallel robots,"ASME Journal of Mechanical Design, Vol. 128, No. 1, pp. 199-206.

[19] Briot, S., Pashkevich,A.,andChablat, D., 2010,“Optimal Technology-Oriented Design of Parallel Robots for HighSpeed Machining Applications," in Proc. 2010 IEEE International Conference on Robotics and Automation (ICRA 2010), pp. 1155-1161.

[20]Miossec, S., and Nouaille, L., "Structural Link Optimization of an Echography Robot," IFToMM World Congress, 2011. 